\title{
Pyrochlore-Supergroup Minerals Nomenclature: An Update
}

\author{
Daniel Atencio * \\ Instituto de Geociências - Universidade de São Paulo, São Paulo, Brazil
}

The general formula of the pyrochlore-supergroup minerals is $A_{2} B_{2} X_{6} Y$. The mineral names are composed of two prefixes and one root name (identical to the name of the group). The first prefix refers to the dominant anion (or cation or $\mathrm{H}_{2} \mathrm{O}$ or vacancy) of the dominant valence at the $Y$-site. The second prefix refers to the dominant cation of the dominant valence [or $\mathrm{H}_{2} \mathrm{O}$ or vacancy] at the $A$-site. Thirty-one pyrochlore-supergroup mineral species are currently distributed into four groups [pyrochlore $(B=\mathrm{Nb}, X=O)$, microlite $(B=\mathrm{Ta}, X=O)$, roméite $\left(B=\mathrm{Sb}^{5+}, X=O\right)$, and elsmoreite $(B=\mathrm{W}, X=O)$ ] and two

\section{OPEN ACCESS}

Edited by:

Sarah C. Finkeldei,

University of California, Irvine,

United States

Reviewed by:

Xiaolong Zhu,

Merck, United States

Brendan Kennedy,

The University of Sydney, Australia

${ }^{*}$ Correspondence: Daniel Atencio datencio@usp.br

Specialty section: This article was submitted to Solid State Chemistry,

a section of the journal

Frontiers in Chemistry

Received: 22 May 2021 Accepted: 06 August 2021 Published: 06 September 2021

Citation:

Atencio D (2021) PyrochloreSupergroup Minerals Nomenclature: An Update.

Front. Chem. 9:713368. doi: $10.3389 /$ fchem. 2021.713368 unassigned members [hydrokenoralstonite $(B=\mathrm{Al}, X=\mathrm{F})$ and fluornatrocoulsellite $(B=$ $\mathrm{Mg}, X=\mathrm{F})$ ]. However, when the new nomenclature system of this supergroup was introduced (2010) only seven mineral species, namely, oxycalciopyrochlore, hydropyrochlore, hydroxykenomicrolite, oxystannomicrolite, oxystibiomicrolite, hydroxycalcioroméite, and hydrokenoelsmoreite, were valid. The seven species belong to the cubic crystal system and space group $F d \overline{3} m$ and $\mathrm{O}$ is predominant in the $X$ structural site. The 24 new mineral species described between 2010 and 2021 are cesiokenopyrochlore, fluorcalciopyrochlore, fluornatropyrochlore, hydrokenopyrochlore, hydroxycalciopyrochlore, hydroxynatropyrochlore, hydroxykenopyrochlore, hydroxymanganopyrochlore, hydroxyplumbopyrochlore, fluorcalciomicrolite, fluornatromicrolite, hydrokenomicrolite, hydroxycalciomicrolite, kenoplumbomicrolite, oxynatromicrolite, oxycalciomicrolite, oxybismutomicrolite, fluorcalcioroméite, hydroxyferroroméite, oxycalcioroméite, oxyplumboroméite, fluornatrocoulsellite, hydrokenoralstonite, and hydroxykenoelsmoreite. Among the new species, hydroxycalciomicrolite belongs to a different space group of the cubic system, i.e., $P 4_{2} 32$. There are also some mineral species that crystallize in the trigonal system. Hydrokenoelsmoreite occurs as $3 C(F d \overline{3} m)$ and $6 R(R \overline{3})$ polytypes. Hydrokenomicrolite occurs as $3 C(F d \overline{3} m)$ and $3 R(R \overline{3} m)$ polytypes, of which the latter corresponds to the discredited "parabariomicrolite." Fluornatrocoulsellite crystallizes as $3 R(R \overline{3} m)$ polytype. Surely there are several new pyrochloresupergroup minerals to be described.

Keywords: pyrochlore supergroup, nomenclature, pyrochlore group, microlite group, elsmoreite group, roméite group 


\section{INTRODUCTION}

The nomenclature system currently valid for the pyrochlore supergroup was introduced by Atencio et al. (2010) to replace the one authored by Hogarth (1977). Subsequently, clarifications (Christy and Atencio, 2013), remarks (Hogarth, 2013), a response to the remarks (Atencio, 2013), and a paper on the incorporation of two minerals already known to the supergroup (Atencio et al., 2017) were published. When the new nomenclature system of this supergroup was introduced (Atencio et al., 2010), only seven mineral species, namely, oxycalciopyrochlore, hydropyrochlore, hydroxykenomicrolite, oxystannomicrolite, oxystibiomicrolite, hydroxycalcioroméite, and hydrokenoelsmoreite, were valid. Between 2010 and 2021, 24 new mineral species were described. The following text describes the nomenclature of pyrochlore-supergroup minerals. The nomenclature system has been updated to include the pyrochlore-supergroup minerals discovered in recent years, whose available information might be difficult for an interested reader to find. The representative minerals from each group are discussed in detail. All known minerals of the supergroup are listed in one place, so this text can be regarded as a kind of a digest of all natural species. There are two aims in compiling this data. The first is to enable a reader to identify both the chemical composition and source of a given mineral and the second is to enable the reader to identify the primary data associated with the mineral.

\section{CRYSTALLOGRAPHY, CHEMISTRY, AND THE NOMENCLATURE SCHEME}

The general formula of the pyrochlore-supergroup minerals is $A_{2} B_{2} X_{6} Y$. In this formula, $A$ typically is a large [8]-coordinated cation with a radius of $\sim 1.0 \AA$ or a vacancy ( $\square$ ) but can also be $\mathrm{H}_{2} \mathrm{O}$. For structural reasons, $A$ can be subdivided into constituents without lone-pair electrons (e.g., $\mathrm{Na}, \mathrm{Ca}$ ), which occupy $16 \mathrm{~d}$ in $F d \overline{3} m$, and stereoactive cations (e.g., $\mathrm{Sb}^{3+}$ ), which occupy less symmetrical positions displaced slightly from $16 \mathrm{~d}$, e.g., $96 \mathrm{~g}$. For the purpose of this nomenclature, no subdivision is made. The $A$-site therefore may host $\mathrm{Na}, \mathrm{Ca}, \mathrm{Ag}, \mathrm{Mn}, \mathrm{Sr}, \mathrm{Ba}, \mathrm{Fe}^{2+}$, $\mathrm{Pb}^{2+}, \mathrm{Sn}^{2+}, \mathrm{Sb}^{3+}, \mathrm{Bi}^{3+}, \mathrm{Y}, \mathrm{Ce}$ (and other REE), $\mathrm{Sc}, \mathrm{U}, \mathrm{Th}, \square$, or $\mathrm{H}_{2} \mathrm{O} . B$ is a [6]-coordinated cation (site $16 \mathrm{c}$ ), typically of high field-strength. This site thus may contain $\mathrm{Ta}, \mathrm{Nb}, \mathrm{Ti}^{4+}, \mathrm{Sb}^{5+}, \mathrm{W}$,

\begin{tabular}{lcc}
\hline TABLE 1 | Mineral groups of the pyrochlore supergroup. & \\
\hline Group & $\boldsymbol{B}$ & $\boldsymbol{X}$ \\
\hline Elsmoreite & $\mathrm{W}^{6+}$ & $\mathrm{O}^{2-}$ \\
Pyrochlore & $\mathrm{Nb}^{5+}$ & $\mathrm{O}^{2-}$ \\
Microlite & $\mathrm{Ta}^{5+}$ & $\mathrm{O}^{2-}$ \\
Roméite & $\mathrm{Sb}^{5+}$ & $\mathrm{O}^{2-}$ \\
Betafite & $\mathrm{Ti}^{4+}$ & $\mathrm{O}^{2-}$ \\
Ralstonite & $\mathrm{Al}^{3+}$ & $\mathrm{F}^{1-}$ \\
Coulsellite & $\mathrm{Mg}^{2+}$ & $\mathrm{F}^{1-}$
\end{tabular}

$\mathrm{B}$ : the dominant cation of the dominant valence at the B-site.

$\mathrm{X}$ : the dominant anion of the dominant valence at the X-site. but also $\mathrm{V}^{5+}, \mathrm{Sn}^{4+}, \mathrm{Zr}, \mathrm{Hf}, \mathrm{Fe}^{3+}, \mathrm{Mg}, \mathrm{Al}$, and $\mathrm{Si} . X$ typically is $\mathrm{O}$ but can include subordinate $\mathrm{OH}$ and $\mathrm{F}$ (site 48f). $Y$ typically is an anion but can also be a vacancy, $\mathrm{H}_{2} \mathrm{O}$, or a very large $(>>1.0 \AA)$ monovalent cation (site 8b). Examples are $\mathrm{OH}^{-}, \mathrm{F}, \mathrm{O}, \square, \mathrm{H}_{2} \mathrm{O}, \mathrm{K}$, $\mathrm{Cs}$, and Rb. Displacements to $96 \mathrm{~g}, 32 \mathrm{e}$, and $192 \mathrm{i}$ positions were also located. See the basis for formula calculation in Atencio et al. (2010). Synthetic pyrochlores have a much more variable chemical composition than natural examples (Subramanian et al., 1983).

The mineral names are composed of two prefixes and one root name (identical to the name of the group). The first prefix refers to the dominant anion (or cation or $\mathrm{H}_{2} \mathrm{O}$ or $\square$ ) of the dominant valence at the $Y$-site. The second prefix refers to the dominant cation of the dominant valence [or $\mathrm{H}_{2} \mathrm{O}$ or $\square$ ] at the $A$-site. Where the first and second prefixes are equal, then only one prefix is applied ("hydropyrochlore," not "hydrohydropyrochlore"). The mineral groups are given in Table $\mathbf{1}$.

As a mineral group consists of two or more minerals (Mills et al., 2009), ralstonite and coulsellite cannot really be considered, for now, as mineral groups. Hydrokenoralstonite and fluornatrocoulsellite should be designated as unassigned members of the pyrochlore supergroup, because there is no other member to allow a group to be established.

Currently, there is no valid betafite-group mineral.

The seven species valid in 2010 belong to the cubic crystal system and space group $F d \overline{3} m$. Among the new species, hydroxycalciomicrolite belongs to a different space group of the cubic system, i.e., $P 4_{2} 32$. There are also some mineral species that crystallize in the trigonal system. Hydrokenoelsmoreite occurs as $3 C(F d \overline{3} m)$ and $6 R(R \overline{3})$ polytypes. Hydrokenomicrolite occurs as $3 C(F d \overline{3} m)$ and $3 R$ $(R \overline{3} m)$ polytypes, of which the latter corresponds to the discredited "parabariomicrolite." Fluornatrocoulsellite crystallizes as $3 R(R \overline{3} m)$ polytype. The symmetry is lowered due to ordering on either A sites or B sites (Ercit et al., 1986; Rouse et al., 1998; Atencio, 2016; Mills et al., 2016; Andrade et al., 2017; Mills et al., 2017). The pyrochlore structure (Figure 1) is an essential building block for other minerals and mineral groups, such as alunite (Goreaud and Raveau, 1980) or pittongite (Grey et al., 2006).

\section{THE GROUPS AND SPECIES}

Table 2 shows the species of the pyrochlore supergroup, except hydrokenoralstonite and fluornatrocoulsellite.

Following that, simplified formulae are given for the pyrochlore species. Note that subordinate components at the $A, B, X$, or $Y$ sites have no nomenclatural significance and any of these could be replaced by "\#”, indicating an unspecified heterovalent species required for charge balance.

\section{PYROCHLORE GROUP}

Oxycalciopyrochlore (Atencio et al., 2010), $\mathrm{Ca}_{2} \mathrm{Nb}_{2} \mathrm{O}_{6} \mathrm{O}, F d \overline{3} m, a$ 10.356(2) $\AA, V 1110.6$ (7) $\AA^{3}$, first described by Černý et al. (1979) 


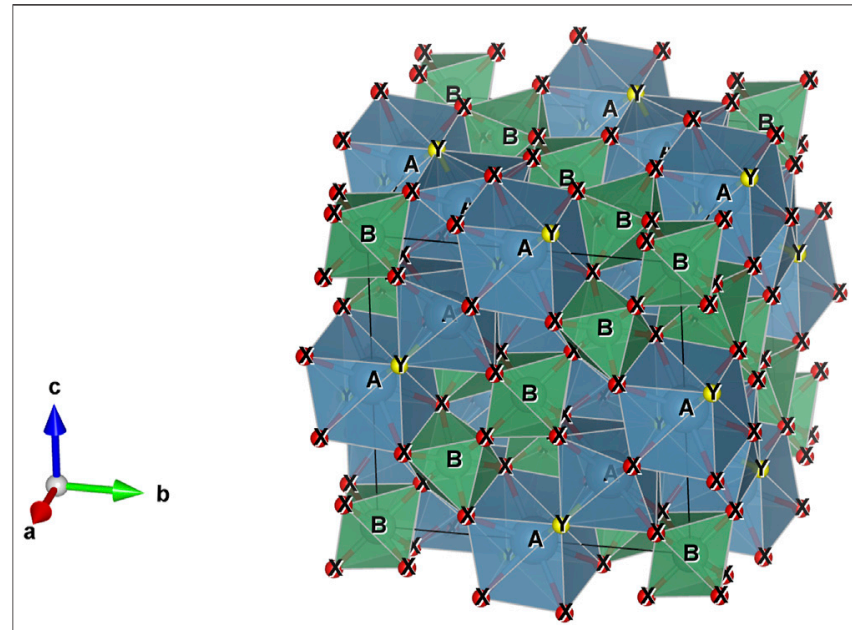

FIGURE 1 | Pyrochlore crystal structure drawn using VESTA 3 (Momma and Izumi, 2011).

as "stibiobetafite." The IMA number was not quoted in the original description of "stibiobetafite." Type locality: Věžná I pegmatite, Věžná, Ždár nad Sázavou District, Vysočina Region, Czech Republic.

Hydropyrochlore (Atencio et al., 2010), $\left(\mathrm{H}_{2} \mathrm{O}\right.$, $\#)_{2} \mathrm{Nb}_{2} \mathrm{O}_{6}\left(\mathrm{H}_{2} \mathrm{O}\right), \quad F d \overline{3} m$, a $10.580 \AA$, $V 1184.29 \AA^{3}$, first described by van Wambeke (1978) as "kalipyrochlore." The IMA number was not quoted in the original description of "kalipyrochlore." Type locality: Lueshe Mine, Bwito, Rutshuru Territory, North Kivu, DR Congo.

Cesiokenopyrochlore (Agakhanov et al., 2021), (口, $\#)_{2} \mathrm{Nb}_{2} \mathrm{O}_{6} \mathrm{Cs}, F d \overline{3} m$, a 10.444(1) $\AA$, $V$ 1139.5(2) $\AA^{3}$. IMA number: 2016-104. Type locality: Tetezantsio pegmatites, Tetezantsio-Andoabatokely Pegmatite Field, Andrembesoa, Betafo, Vakinankaratra, Madagascar.

Fluorcalciopyrochlore (Li et al., 2016), (Ca, \#) ${ }_{2} \mathrm{Nb}_{2} \mathrm{O}_{6} \mathrm{~F}$, $F d \overline{3} m$, a 10.4164(9) $\AA, V 1130.19 \AA^{3}$. IMA number: 2013-055. Type locality: Bayan Obo deposit (Bayun-Obo deposit; Baiyunebo deposit), Bayan Obo, Bayan Obo mining district, Baotou City (Baotou Prefecture), Inner Mongolia, China.

Fluornatropyrochlore (Jingwu et al., 2015), $(\mathrm{Na}, \#)_{2} \mathrm{Nb}_{2} \mathrm{O}_{6} \mathrm{~F}$, $F d \overline{3} m$, a 10. 5,053(10) А, V $1159.4 \AA^{3}$. IMA number: 2013-056. Type locality: Boziguoer intrusion, Baicheng Co. (Bay Co.), Akesu Prefecture (Aksu Prefecture; Aqsu Prefecture), Xinjiang, China.

Hydrokenopyrochlore (Biagioni et al., 2018), (口, $\#)_{2} \mathrm{Nb}_{2} \mathrm{O}_{6}\left(\mathrm{H}_{2} \mathrm{O}\right), F d \overline{3} m$, a $10.4887(8) \AA ⿻$, $V 1153.9 \AA^{3}$. IMA number: 2017-005. Type locality: Antandrokomby pegmatite, Manandona Valley, Sahatsiho Ambohimanjaka, Ambositra, Amoron'i Mania, Madagascar.

Hydroxycalciopyrochlore (Yang et al., 2014), (Ca, $\#)_{2} \mathrm{Nb}_{2} \mathrm{O}_{6}(\mathrm{OH}), \quad F d \overline{3} m$, a 10.381(4) $\AA, \quad V 1118.7 \AA^{3}$. IMA number: 2011-026. Type locality: Maoniuping Mine, Mianning County, Liangshan Yi, Sichuan, China.

Hydroxynatropyrochlore (Ivanyuk et al., 2019), (Na, $\#)_{2} \mathrm{Nb}_{2} \mathrm{O}_{6}(\mathrm{OH}), \quad F d \overline{3} m$, a $10.3276(5) \AA ⿻, V 1101.5 \AA^{3}$. IMA number: 2017-074. Type locality: Phoscorite-carbonatite pipe, Kovdor Massif, Murmansk Oblast, Russia.
TABLE 2 | Mineral species of the pyrochlore $(P)$, microlite $(M)$, roméite $(R)$ elsmoreite $(E)$, and betafite $(B)$ groups. Blue: already valid in 2010; red: expected in 2010 and described later; brown: expected in 2010 but not yet described; green: not foreseen in 2010 but described later.

\begin{tabular}{lcccccc}
\hline $\boldsymbol{A} \downarrow \boldsymbol{Y} \rightarrow$ & $\mathbf{O}$ & OH & $\mathbf{F}$ & $\square$ & $\mathbf{H}_{\mathbf{2}} \mathbf{O}$ & Cs \\
\hline$\square$ & & $\mathrm{PME}$ & $\mathrm{P}$ & & $\mathrm{PME}$ & $\mathrm{P}$ \\
$\mathrm{H}_{2} \mathrm{O}$ & & & & \\
$\mathrm{Na}$ & $\mathrm{MP}$ & $\mathrm{P}$ & $\mathrm{PMR}$ & & \\
$\mathrm{Ca}$ & $\mathrm{PMR} \mathrm{B}$ & $\mathrm{PMR}$ & $\mathrm{PMR}$ & & \\
$\mathrm{Sr}$ & & & $\mathrm{P}$ & & \\
$\mathrm{Fe}^{2+}$ & & $\mathrm{R}$ & & \\
$\mathrm{Mn}^{2+}$ & & $\mathrm{P}$ & & \\
$\mathrm{Sn}^{2+}$ & $\mathrm{M}$ & & & \\
$\mathrm{Pb}^{2+}$ & $\mathrm{PR}$ & $\mathrm{P}$ & & $\mathrm{PM}$ & \\
$\mathrm{Sb}^{3+}$ & $\mathrm{M}$ & & & \\
$\mathrm{Bi}$ & $\mathrm{M}$ & & & \\
$\mathrm{Y}$ & $\mathrm{P}$ & & & \\
$\mathrm{U}$ & $\mathrm{B}$ & & &
\end{tabular}

Hydroxykenopyrochlore (Miyawaki et al., 2017, pending publication), (口, \#) ${ }_{2} \mathrm{Nb}_{2} \mathrm{O}_{6}(\mathrm{OH}), \quad F d \overline{3} m$, a $10.590(5) \AA, V$ $1187.65 \AA^{3}$. IMA number: 2017-030a. Type locality: Araxá mine, Araxá complex, Barreiro, Araxá, Minas Gerais, Brazil.

Hydroxymanganopyrochlore (Chukanov et al., 2013), $\left(\mathrm{Mn}^{2+}\right.$, $\#)_{2} \mathrm{Nb}_{2} \mathrm{O}_{6}(\mathrm{OH}), F d \overline{3} m$, a 10.2523(2) $\AA, V 1077.62 \AA^{3}$. IMA number: 2012-005. Type locality: In den Dellen quarries, Mendig, Mendig, Mayen-Koblenz District, RhinelandPalatinate, Germany.

Hydroxyplumbopyrochlore (Li et al., 2020), (Pb, \#) ${ }_{2} \mathrm{Nb}_{2} \mathrm{O}_{6}$ $(\mathrm{OH}), F d \overline{3} m$, a 10.558 (2) $\AA, V 1176.91 \AA^{3}$. IMA number: 2018 145. Type locality: Jabal Sayid mine (Jabal Sayid Cu-Zn deposit), Medina Region, Saudi Arabia.

"Fluorstrontiopyrochlore" (Atencio et al., 2010), (Sr, $\#)_{2} \mathrm{Nb}_{2} \mathrm{O}_{6} \mathrm{~F}$, a possible new species, analysis published (Franchini et al., 2005). Occurrence: Jasimampa prospect, Ojo de Agua Department, Santiago del Estero Province, Argentina.

"Fluorkenopyrochlore" (Atencio et al., 2010), ( $\square$, \#) ${ }_{2} \mathrm{Nb}_{2} \mathrm{O}_{6} \mathrm{~F}$, a possible new species, analyses published (Kartashov et al., 1998; Schmitt et al., 2002). Occurrences: Khaldzan Buragtag massif, Myangad District, Khovd Province, Mongolia; Amis Complex, Brandberg Complex, Brandberg Area, Dâures Constituency, Erongo Region, Greenland.

“Oxynatropyrochlore" (Atencio et al., 2010), (Na, $\#)_{2} \mathrm{Nb}_{2} \mathrm{O}_{6} \mathrm{O}$, a possible new species, analyses published (Hogarth and Horne 1989; Knudsen 1989; Chukanov et al., 1999). Occurrences: Locality 2, Ndale Area, Fort Portal, Kabarole, Western Region, Uganda; Qaqqaarsuk, Maniitsoq, Maniitsoq Island, Qeqqata, Greenland; Mika pegmatite, Rangkul' Highlands, Gorno-Badakhshan, Tajikistan.

“Oxyplumbopyrochlore” (Atencio et al., 2010), $\mathrm{Pb}_{2} \mathrm{Nb}_{2} \mathrm{O}_{6} \mathrm{O}$, a possible new species, analysis published (Voloshin and Pakhomovskiy, 1986). Occurrence: Kola Peninsula, Murmansk Oblast, Russia.

“Oxyyttropyrochlore-(Y)” (Atencio et al., 2010), (Y, $\#)_{2} \mathrm{Nb}_{2} \mathrm{O}_{6} \mathrm{O}$, a possible new species, analysis published (Tindle and Breaks, 1998). Occurrence: Separation Rapids Lithium Project (Separation Lake area), Kenora District, Ontario, Canada. 
“Kenoplumbopyrochlore” (Atencio et al., 2010), (Pb, \#) $\mathrm{Nb}_{2} \mathrm{O}_{6} \square$, a possible new species, analysis published (Voloshin and Pakhomovskiy, 1986). Occurrence: Ploskaya Mt, Western Keivy Massif, Keivy Mountains, Lovozersky District, Murmansk Oblast, Russia.

\section{MICROLITE GROUP}

Hydroxykenomicrolite (Atencio et al., 2010), ( $\square$, \#) ${ }_{2} \mathrm{Ta}_{2} \mathrm{O}_{6}$ (OH), Fd $\overline{3} m, a 10.526$ (5) $\AA, V 1166.244 \AA^{3}$, first described by Voloshin et al. (1981) as "cesstibtantite." The IMA number was not quoted in the original description of "cesstibtantite." Type locality: Vasin-Myl'k Mt, Voron'i Tundry, Murmansk Oblast, Russia.

Oxystannomicrolite (Atencio et al., 2010), $\mathrm{Sn}_{2} \mathrm{Ta}_{2} \mathrm{O}_{6} \mathrm{O}, F d \overline{3} m$, a $10.470 \AA, V 1147.73 \AA^{3}$, first described by Vorma and Siivola (1967) as "sukulaite." The IMA number was not quoted in the original description of "sukulaite." Type locality: Sukula Pegmatites, Tammela, Tavastia Proper, Finland.

Oxystibiomicrolite (Atencio et al., 2010), $\left(\mathrm{Sb}^{3+}, \#\right)_{2} \mathrm{Ta}_{2} \mathrm{O}_{6} \mathrm{O}$, $F d \overline{3} m, a 10.455(2) \AA, V 1142.80 \AA^{3}$, first described by Groat et al. (1987) as "stibiomicrolite." The IMA number was not quoted in the original description of "stibiomicrolite." Type locality: Varuträsk, Skellefteå, Västerbotten County, Sweden.

Fluorcalciomicrolite (Andrade et al., 2013a), $\left(\mathrm{Ca}^{2+}, \#\right)_{2}$ $\mathrm{Ta}_{2} \mathrm{O}_{6} \mathrm{~F}, F d \overline{3} m$, a 10.4191 (6) $\AA, V 1131.07 \AA^{3}$. IMA number: 2012-036. Type locality: Volta Grande mine (Mibra mine), Nazareno, Minas Gerais, Brazil.

Fluornatromicrolite (Witzke et al., 2011), (Na, \#) $)_{2} \mathrm{Ta}_{2} \mathrm{O}_{6} \mathrm{~F}$, $F d \overline{3} m$, a 10.4451 (2) $\AA, V 1139.56 \AA^{3}$. IMA number: 1998-018. Type locality: Quixaba pegmatite, Quixaba, Frei Martinho, Paraíba, Brazil.

Hydrokenomicrolite (Andrade et al., 2013b; Atencio, 2016), $(\square, \#)_{2} \mathrm{Ta}_{2} \mathrm{O}_{6}\left(\mathrm{H}_{2} \mathrm{O}\right)$. Hydrokenomicrolite-3C polytype: Cubic, $F d \overline{3} m$, a 10.454(1) A. $V 1142.5$ (2) $\AA^{3}$. Hydrokenomicrolite-3R polytype: Trigonal, $R \overline{3} m$, a 7.4290(6), c 18.505 (2) $\AA, V 884.5$ (1) $\AA^{3}$. IMA Numbers: hydrokenomicrolite (hydrokenomicrolite3C) 2011-103; "parabariomicrolite" (hydrokenomicrolite-3R): 84-3. Type localities: Hydrokenomicrolite-3C (described as hydrokenomicrolite by Andrade et al., 2013b), Volta Grande pegmatite, Nazareno, Minas Gerais, Brazil. Hydrokenomicrolite-3R (formerly described as "parabariomicrolite" by Ercit et al., 1986), Alto do Giz pegmatite, Equador Co., Rio Grande do Norte, Brazil.

Hydroxycalciomicrolite (Andrade et al., 2017), $\left(\mathrm{Ca}^{2+}, \#\right)_{2}$ $\mathrm{Ta}_{2} \mathrm{O}_{6}(\mathrm{OH}), \quad P 4_{2} 32$, a 10.4205(8) $\AA \mathrm{V} 1131.53 \AA^{3}$. The first pyrochlore-supergroup mineral with long range ordering of $\mathrm{Ca}$ and $\square$ on the $A$ sites, that invokes reduction of symmetry. IMA number: 2013-073. Type locality: Volta Grande mine (Mibra mine), Nazareno, Minas Gerais, Brazil.

Kenoplumbomicrolite (Atencio et al., 2018), ( $\mathrm{Pb}, \#)_{2} \mathrm{Ta}_{2} \mathrm{O}_{6} \square$, $P 4_{2} 32$, a 10.575 (5) $\AA V 1182.6 \AA^{3}$. IMA number: 2015-007-a. Type locality: Ploskaya Mt, Western Keivy Massif, Keivy Mountains, Lovozersky District, Murmansk Oblast, Russia.

Oxynatromicrolite (Fan et al., 2016), $(\mathrm{Na}, \#)_{2} \mathrm{Ta}_{2} \mathrm{O}_{6} \mathrm{O}, \mathrm{Fd} \overline{3} \mathrm{~m}$, a 10.420(6) $\AA, V 1131.4 \AA^{3}$. IMA number: 2013-063. Type locality: Pegmatite vein no. 309, Guanpo pegmatite field, Guanpo, Lushi County, Sanmenxia, Henan, China.

Oxycalciomicrolite (Menezes da Silva et al., 2020), $\mathrm{Ca}_{2} \mathrm{Ta}_{2} \mathrm{O}_{6} \mathrm{O}, F d \overline{3} m$, a 10.4325 (4) $\AA, V$ 1135.46(14) $\AA^{3}$. IMA number: 2019-110. Type locality: Fumal pegmatite, Nazareno, Minas Gerais, Brazil.

Oxybismutomicrolite (Kasatkin et al., 2020), (Bi, \#) $)_{2} \mathrm{Ta}_{2} \mathrm{O}_{6} \mathrm{O}$, $F d \overline{3} m$, a 10.475 (1) $\AA, V 1149.38 \AA^{3}$. IMA number: 2019-047. Type locality: Solnechnaya pegmatite, Malkhan pegmatite field (Malchan; "Malechansk"), Krasnyi Chikoy, Zabaykalsky Krai, Russia.

"Hydromicrolite" (Atencio et al., 2010), ( $\left.\mathrm{H}_{2} \mathrm{O}, \#\right)_{2}$ $\mathrm{Nb}_{2} \mathrm{O}_{6}\left(\mathrm{H}_{2} \mathrm{O}\right)$, a possible new species, analysis published (Andrade, 2007). Occurrence: Volta Grande mine (Mibra mine), Nazareno, Minas Gerais, Brazil.

\section{ROMÉITE GROUP}

Hydroxycalcioroméite (Atencio et al., 2010), (Ca, $\#)_{2} \mathrm{Sb}_{2}^{5+} \mathrm{O}_{6}(\mathrm{OH}), \quad F d \overline{3} m$, a $10.264 \AA, \quad V 1081.31 \AA^{3}$, first described by Hussak and Prior (1895) as "lewisite." IMA number: a pre-IMA mineral. Type locality: Tripuí (Tripuhy), Ouro Preto, Minas Gerais, Brazil.

Fluorcalcioroméite (Atencio et al., 2013), (Ca, \#) $)_{2} \mathrm{Sb}_{2}^{5+} \mathrm{O}_{6} \mathrm{~F}$, $F d \overline{3} m$, a 10.2987 (8) Å, $V 1092.31 \AA^{3}$. IMA number: 2012-093. Type locality: Starlera Mine, Starlera Valley, Ferrera, Viamala Region, Grisons, Switzerland.

Hydroxyferroroméite (Mills et al., 2017a), (Fe ${ }^{2+}$, $\#)_{2} \mathrm{Sb}_{2}^{5+} \mathrm{O}_{6}(\mathrm{OH}), \quad F d \overline{3} m$, a 10.25 (3) $\AA, \quad V \quad 1077 \AA^{3}$. IMA number: 2016-006. Type locality: Correc d'en Llinassos (Ravin d'en Llinassous), Oms, Céret, Pyrénées-Orientales, Occitanie, France.

Oxycalcioroméite (Biagioni et al., 2013), $\mathrm{Ca}_{2} \mathrm{Sb}_{2}^{5+} \mathrm{O}_{6} \mathrm{O}, F d \overline{3} m$, a 10.3042 (7) $\AA, V 1094.06 \AA^{3}$. IMA number: 2012-022. Type locality: Buca della Vena Mine, Ponte Stazzemese, Stazzema, Lucca Province, Tuscany, Italy.

Oxyplumboroméite (Hålenius and Bosi, 2013), $\mathrm{Pb}_{2} \mathrm{Sb}_{2}^{5+} \mathrm{O}_{6} \mathrm{O}$, $F d \overline{3} m$, a 10.3783 (6) $\AA, V 1117.84 \AA^{3}$. IMA number: 2013-042. Type locality: Harstigen Mine, Pajsberg, Persberg ore district, Filipstad, Värmland County, Sweden.

"Fluornatroroméite" (Atencio et al., 2010), (Na, \#) $)_{2} \mathrm{Sb}_{2}^{5+} \mathrm{O}_{6} \mathrm{~F}$, a possible new species, crystal structure determined (Matsubara et al., 1996). Occurrence: Gozaisho mine, Iwaki, Japan.

\section{ELSMOREITE GROUP}

Hydrokenoelsmoreite (Atencio et al., 2010), $\square_{2} \mathrm{~W}_{2} \mathrm{O}_{6}\left(\mathrm{H}_{2} \mathrm{O}\right)$, first described by Williams et al. (2005) as "elsmoreite". Hydrokenoelsmoreite-3C polytype: Cubic, $F d \overline{3} m$, a 10.3065(3) $\AA$. $V 1094.80 \AA^{3}$. Hydrokenoelsmoreite- $6 R$ polytype: Trigonal, $R \overline{3}, a$ 7.2882(2), c 35.7056(14) $\AA, V 1642.51 \AA^{3}$. IMA Numbers: “elsmoreite" (hydrokenoelsmoreite-3C) 2003-059. Type localities: Hydrokenoelsmoreite-3C (described as elsmoreite by Williams et al., 2005): Elsmore Tin Mine (Elsmore Tin lodes), Elsmore, Gough Co., New South Wales, Australia; 
hydrokenoelsmoreite-3C and hydrokenoelsmoreite-6R (formerly "ferritungstite") from Hemerdon mine (now Drakelands mine) in Devon, United Kingdom (Mills et al., 2016).

Hydroxykenoelsmoreite (Mills et al., 2017b), (口, \#) $\mathrm{W}_{2} \mathrm{O}_{6}(\mathrm{OH})$, Trigonal, $R \overline{3}$, a 7.313(2), c 17.863(7) $\AA$, $V$ 827(1) $\AA^{3}$. IMA number: 2016-056. Type locality: Masaka gold mine, Muyinga Province, Burundi.

\section{UNASSIGNED MEMBERS}

Fluornatrocoulsellite (Atencio et al., 2017), (Na, \#) $)_{2} \mathrm{Mg}_{2} \mathrm{~F}_{6} \mathrm{~F}$, $R \overline{3} m$, a 7.1620(1), c 17.5972(3) $\AA, V 781.7049 \AA^{3}$, first described by Birch et al. (2009) as "cousellite." IMA number: 2009-046 (coulsellite). Type locality: Mt Cleveland Mine, Luina, Heazlewood district, Waratah-Wynyard municipality, Tasmania, Australia.

Hydrokenoralstonite (Atencio et al., 2017), $\square_{2} \mathrm{Al}_{2} \mathrm{~F}_{6}\left(\mathrm{H}_{2} \mathrm{O}\right.$ ), $F d \overline{3} m, a$ 9.91(4) Å. $V$ 973.24 $\AA^{3}$, first described by Brush (1871) as "ralstonite." IMA number: pre-IMA mineral. Type locality: Ivigtut Mine, Arsuk Fjord, Sermersooq, Greenland.

\section{BETAFITE GROUP}

“Oxycalciobetafite” (Atencio et al., 2010), (Ca, $\#)_{2} \mathrm{Ti}_{2} \mathrm{O}_{6} \mathrm{O}$, a possible new species, analysis published (Meyer and Yang,

\section{REFERENCES}

Agakhanov, A. A., Kasatkin, A. V., Britvin, S. N., Siidra, O. I., Pautov, L. A., Pekov, I. V., et al. (2021). Cesiokenopyrochlore, the First Natural Niobate with an Inverse Pyrochlore Structure. Can. Mineral. 59, 149-157. doi:10.3749/canmin.2000056

Andrade, M. B., Atencio, D., Chukanov, N. V., and Ellena, J. (2013b). Hydrokenomicrolite, $\left(\square, \mathrm{H}_{2} \mathrm{O}\right)_{2} \mathrm{Ta}_{2}(\mathrm{O}, \mathrm{OH})_{6}\left(\mathrm{H}_{2} \mathrm{O}\right)$, a New Microlite-Group mineral from Volta Grande Pegmatite, Nazareno, Minas Gerais, Brazil. Am. Mineral. 98, 292-296. doi:10.2138/am.2013.4186

Andrade, M. B., Atencio, D., Persiano, A. I. C., and Ellena, J. (2013a). Fluorcalciomicrolite, $(\mathrm{Ca}, \mathrm{Na}, \square)_{2} \mathrm{Ta}_{2} \mathrm{O}_{6} \mathrm{~F}$, a New Microlite-Group mineral from Volta Grande Pegmatite, Nazareno, Minas Gerais, Brazil. Mineral. Mag. 77 (7), 2989-2996. doi:10.1180/minmag.2013.077.7.08

Andrade, M. B. (2007). "Estudo cristaloquímico de minerais do grupo do pirocloro no Brasil,”. Tese de Doutorado (São Paulo: Instituto de Geociências), 207. doi:10.11606/T.44.2007.tde-30072007-165039

Andrade, M. B., Yang, H., Atencio, D., Downs, R. T., Chukanov, N. V., LeméeCailleau, M. H., et al. (2017). Hydroxycalciomicrolite, $\mathrm{Ca}_{1.5} \mathrm{Ta}_{2} \mathrm{O}_{6}(\mathrm{OH})$, a New Member of the Microlite Group from Volta Grande Pegmatite, Nazareno, Minas Gerais, Brazil. Mineral. Mag. 81, 555-564. doi:10.1180/minmag.2016.080.116

Atencio, D., Andrade, M. B., Bastos Neto, A. C., and Pereira, V. P. (2017). Ralstonite Renamed Hydrokenoralstonite, Coulsellite Renamed Fluornatrocoulsellite, and Their Incorporation into the Pyrochlore Supergroup. Can. Mineral. 55 (1), 115-120. doi:10.3749/canmin.1600056

Atencio, D., Andrade, M. B., Bindi, L., Bonazzi, P., Zoppi, M., Stanley, C. J., et al. (2018). Kenoplumbomicrolite, $(\mathrm{Pb}, \square){ }_{2} \mathrm{Ta}_{2} \mathrm{O}_{6}[\square,(\mathrm{OH}), \mathrm{O}]$, a New mineral from Ploskaya, Kola Peninsula, Russia. Mineral. Mag. 82, 1049-1055. doi:10.1180/minmag.2017.081.082

Atencio, D., Andrade, M. B., Christy, A. G., Gieré, R., and Kartashov, P. M. (2010). The Pyrochlore Supergroup of Minerals: Nomenclature. Can. Mineral. 48, 673-698. doi:10.3749/canmin.48.3.673

Atencio, D., Ciriotti, M. E., and Andrade, M. B. (2013). Fluorcalcioroméite, $(\mathrm{Ca}, \mathrm{Na})_{2} \mathrm{Sb}_{2}{ }^{5+}(\mathrm{O}, \mathrm{OH})_{6} \mathrm{~F}$, a New Roméite-Group mineral from Starlera Mine, Ferrera, Grischun, Switzerland: Description and crystal Structure. Mineral. Mag. 77, 467-473. doi:10.1180/minmag.2013.077.4.06
1988). Occurrence: Fra Mauro Base (Apollo 14 landing site), Fra Mauro Highlands, The Moon.

“Oxyuranobetafite" (Atencio et al., 2010), (U, \#) ${ }_{2} \mathrm{Ti}_{2} \mathrm{O}_{6} \mathrm{O}$, a possible new species, analysis published (Mokhov et al., 2008). Occurrence: Luna 24 landing site, Mare Crisium, The Moon.

\section{DATA AVAILABILITY STATEMENT}

The original contributions presented in the study are included in the article/supplementary material; further inquiries can be directed to the corresponding author.

\section{AUTHOR CONTRIBUTIONS}

The author confirms being the sole contributor of this work and has approved it for publication.

\section{ACKNOWLEDGMENTS}

The author acknowledges FAPESP (Fundação de Amparo à Pesquisa do Estado de São Paulo) for financial support (process 2019/23498-0) and $\mathrm{CNPq}$ for research productivity scholarship (process 303431/2019-9).

Atencio, D. (2016). Parabariomicrolite Discredited as Identical to hydrokenomicrolite-3R. Mineral. Mag. 80, 923-924. doi:10.1180/ minmag.2016.080.129

Atencio, D. (2013). The Pyrochlore Supergroup: Remarks on Nomenclature Response. Can. Mineral. 51 (5), 803-804. doi:10.3749/canmin.51.5.803

Biagioni, C., Meisser, N., Nestola, F., Pasero, M., Robyr, M., Roth, P., et al. (2018). Hydrokenopyrochlore, $(\square, \#)_{2} \mathrm{Nb}_{2} \mathrm{O}_{6} \cdot \mathrm{H}_{2} \mathrm{O}$, a New Species of the Pyrochlore Supergroup from the Sahatany Pegmatite Field, Antananarivo Province, Madagascar. ejm 30, 869-876. doi:10.1127/ejm/2018/0030-2761

Biagioni, C., Orlandi, P., Nestola, F., and Bianchin, S. (2013). Oxycalcioroméite, Ca2Sb2O6O, from Buca Della Vena Mine, Apuan Alps, Tuscany, Italy: a New Member of the Pyrochlore Supergroup. Mineral. Mag. 77, 3027-3037. doi:10.1180/minmag.2013.077.7.12

Birch, W. D., Grey, I. E., Mumme, W. G., and Pring, A. (2009). Coulsellite, a New mineral from the Cleveland Mine, Luina, Tasmania. Aust. J. Mineralogy 15, 21-24.

Brush, G. J. (1871). On Ralstonite, a New Fluoride from Arksut Fiord [Greenland]. Am. J. Sci. s3-2, 30-31. doi:10.2475/ajs.s3-2.7.30

Černý, P., Hawthorne, F. C., Laflamme, J. H. G., and Hinthorne, J. R. (1979). Stibiobetafite, a New Member of the Pyrochlore Group from Vežná, Czechoslovakia. Can. Mineral. 17, 583-588.

Christy, A. G., and Atencio, D. (2013). Clarification of Status of Species in the Pyrochlore Supergroup. Mineral. Mag. 77 (1), 13-20. doi:10.1180/ minmag.2013.077.1.02

Chukanov, N. V., Blass, G., Zubkova, N. V., Pekov, I. V., Pushcharovskii, D. Y., and Prinz, H. (2013). Hydroxymanganopyrochlore: A New mineral from the Eifel Volcanic Region, Germany. Dokl. Earth Sc. 449 (1), 342-345. doi:10.1134/s1028334x13030100

Chukanov, N. V., Skrigitil, A. M., Kuzmina, O. V., and Zadov, A. E. (1999). Bismutopyrochlore $(\mathrm{Bi}, \mathrm{U}, \mathrm{Ca}, \mathrm{Pb})_{1+\mathrm{x}}(\mathrm{Nb}, \mathrm{Ta})_{2} \mathrm{O}_{6}(\mathrm{OH}) \cdot n \mathrm{H}_{2} \mathrm{O}-\mathrm{a}$ New mineral from the Mika Pegmatite Vein (Eastern Pamirs). Zapiski Vsesoyuznoye Mineralogichestogo Obshchestvo 128 (4), 36-41.

Ercit, T. S., Hawthorne, F. C., and Černy, P. (1986). Parabariomicrolite, a New Species and its Structural Relationship to the Pyrochlore Group. Can. Mineral. 24, 655-663.

Fan, G., Ge, X., Li, G., Yu, A., and Shen, G. (2016). Oxynatromicrolite, $(\mathrm{Na}, \mathrm{Ca}, \mathrm{U})_{2} \mathrm{Ta}_{2} \mathrm{O}_{6}(\mathrm{O}, \mathrm{F})$, a New Member of the Pyrochlore Supergroup from Guanpo, Henan Province, China. Mineralogical Mag. 81 (4), 743-751. 
Franchini, M., Lira, R., Meinert, L., Ríos, F. J., Poklepovic, M. F., Impiccini, A., et al. (2005). Na-Fe-Ca Alteration and LREE (Th-Nb) Mineralization in marble and granitoids of Sierra de Sumampa, Santiago del Estero, Argentina. Econ. Geology 100, 733-764. doi:10.2113/100.4.733

Goreaud, M., and Raveau, B. (1980). Alunite and Crandallite: a Structure Derived from that of Pyrochlore. Am. Mineral. 65, 953-956.

Grey, I. E., Birch, W. D., Bougerol, C., and Mills, S. J. (2006). Unit-cell Intergrowth of Pyrochlore and Hexagonal Tungsten Bronze Structures in Secondary Tungsten Minerals. J. Solid State. Chem. 179, 3860-3869. doi:10.1016/j.jssc.2006.08.030

Groat, L. A., ČErný, P., and Ercit, T. S. (1987). Reinstatement of Stibiomicrolite as a Valid Species. Geologiska Föreningen i Stockholm Förhandlingar 109, 105-109. doi:10.1080/11035898709453757

Hålenius, U., and Bosi, F. (2013). Oxyplumboroméite, Pb2Sb2O7, a New mineral Species of the Pyrochlore Supergroup from Harstigen Mine, Värmland, Sweden. Mineral. Mag. 77, 2931-2939. doi:10.1180/minmag.2013.077.7.04

Hogarth, D. D. (1977). Classification and Nomenclature of the Pyrochlore Group. Am. Mineral. 62, 403-410.

Hogarth, D. D., and Horne, J. E. T. (1989). Non-metamict Uranoan Pyrochlore and Uranpyrochlore from Tuff Near Ndale, Fort Portal Area, Uganda. Mineral. Mag. 53, 257-262. doi:10.1180/minmag.1989.053.370.14

Hogarth, D. D. (2013). The Pyrochlore Group: Remarks on Nomenclature. Can. Mineral. 51 (5), 801. doi:10.3749/canmin.51.5.801

Hussak, E., and Prior, G. T. (1895). Lewisite and Zirkelite, Two New Brazilian Minerals. Mineral. Mag. J. Mineral. Soc. 11, 80-88. doi:10.1180/minmag.1895.011.50.05

Ivanyuk, G. Y., Yakovenchuk, V. N., Panikorovskii, T. L., Konoplyova, N., Pakhomovsky, Y. A., Bazai, A. V., et al. (2019). Hydroxynatropyrochlore, $(\mathrm{Na}, \mathrm{Ca}, \mathrm{Ce})_{2} \mathrm{Nb}_{2} \mathrm{O}_{6}(\mathrm{OH})$, a New Member of the Pyrochlore Group from the Kovdor Phoscorite-Carbonatite Pipe, Kola Peninsula, Russia. MinMag. 83 (1), 107-113. doi:10.1180/minmag.2017.081.102

Jingwu, Y., Li, G., Guangming, Y., Ge, X., Xu, H., and Wang, J. (2015). Fluornatropyrochlore, a New Pyrochlore Supergroup mineral from the Boziguoer Rare Earth Element deposit, Baicheng County, Akesu, Xinjiang, China. Can. Mineral. 53, 455-460.

Kartashov, P. M., Mokhov, A. V., and Kovalenko, V. I. (1998). Rare Earth SrPyrochlore from Western Mongolia: The First Find in Association with Alkalic Granites. Doklady Earth Sci. 359, 510-513.

Kasatkin, A. V., Britvin, S. N., Peretyazhko, I. S., Chukanov, N. V., Škoda, R., and Agakhanov, A. A. (2020). Oxybismutomicrolite, a New Pyrochlore-Supergroup mineral from the Malkhan Pegmatite Field, Central Transbaikalia, Russia. MinMag. 84, 444-454. doi:10.1180/mgm.2020.25

Knudsen, C. (1989). "Pyrochlore Group Minerals from the Qaqarssuk Carbonatite Complex," in Lanthanides, Tantalum and Niobium. Editors P. Möller, P. Černý, and F. Saupe (Berlin: Springer-Verlag). doi:10.1007/978-3-642-87262-4_3

Li, G., Yang, G., Lu, F., Xiong, M., Ge, X., Pan, B., et al. (2016). Fluorcalciopyrochlore, a New mineral Species from Bayan Obo, Inner Mongolia, P.R. China. Can. Mineral. 54, 1285-1291.

Li, T., Li, Z., Fan, G., Fan, H., Zhong, J., Jahdali, N. S., et al. (2020). Hydroxyplumbopyrochlore, (Pb1.5, $\square 0.5) \mathrm{Nb}_{2} \mathrm{O}_{6}(\mathrm{OH})$, a New Member of the Pyrochlore Group from Jabal Sayid, Saudi Arabia. MinMag. 84 (5), 785-790. doi:10.1180/mgm.2020.69

Matsubara, S., Kato, A., Shimizu, M., Sekiuchi, K., and Suzuki, Y. (1996). Romeite from the Gozaisho Mine, Iwaki, Japan. Mineralogical J. 18 (4), 155-160. doi:10.2465/minerj.18.155

Menezes da Silva, V. H. R., Ávila, C. A., Neumann, R., Faulstich, F. R. L., Alves, F. E. A., Almeida, F. B., Cidade, T. P., and Sousa, S. S. C. G. (2020). Oxycalciomicrolite, $(\mathrm{Ca}, \mathrm{Na})_{2}(\mathrm{Ta}, \mathrm{Nb}, \mathrm{Ti})_{2} \mathrm{O}_{6}(\mathrm{O}, \mathrm{F})$, a new member of the microlite group (pyrochlore supergroup) from the Paleoproterozoic São João del Rei Pegmatite Province, Minas Gerais state, Brazil. MinMag. 84, 854-858. doi:10.1180/mgm.2020.74

Meyer, C., and Yang, S. V. (1988). Tungsten-bearing Yttrobetafite in Lunar Granophyre. Am. Mineral. 73, 1420-1425.

Mills, S. J., Christy, A. G., Kampf, A. R., Birch, W. D., and Kasatkin, A. (2017b). Hydroxykenoelsmoreite, the First New mineral from the Republic of Burundi. ejm 29, 491-497. doi:10.1127/ejm/2017/0029-2618

Mills, S. J., Christy, A. G., Rumsey, M. S., Spratt, J., Bittarello, E., Favreau, G., et al. (2017a). Hydroxyferroroméite, a New Secondary Weathering mineral from Oms, France. ejm 29, 307-314. doi:10.1127/ejm/2017/0029-2594

Mills, S. J., Christy, A. G., Rumsey, M. S., and Spratt, J. (2016). The crystal Chemistry of Elsmoreite from the Hemerdon (Drakelands) Mine, UK:
hydrokenoelsmoreite-3C and hydrokenoelsmoreite-6R. Mineral. Mag. 80, 1195-1203. doi:10.1180/minmag.2016.080.058

Mills, S. J., Hatert, F., Nickel, E. H., and Ferraris, G. (2009). The Standardisation of mineral Group Hierarchies: Application to Recent Nomenclature Proposals. ejm 21, 1073-1080. doi:10.1127/0935-1221/ 2009/0021-1994

Miyawaki, R., Momma, K., Matsubara, S., Sano, T., Shigeoka, M., and Horiuchi, H. (2017). Hydroxykenopyrochlore, IMA 2017-030a, CNMNC Newsletter No. 39. Mineralogical Mag. 81, 1279-1286. October 2017, page 1285.

Mokhov, A. V., Kartashov, P. M., Bogatikov, O. A., Ashikhmina, N. A., Magazina, L. O., and Koporulina, E. V. (2008). Fluorite, Hatchettolite, Calcium Sulfate, and bastnasite-(Ce) in the Lunar Regolith from Mare Crisium. Dokl. Earth Sc. 422 (1), 1178-1180. doi:10.1134/s1028334x08070416

Momma, K., and Izumi, F. (2011). VESTA 3for Three-Dimensional Visualization of crystal, Volumetric and Morphology Data. J. Appl. Cryst. 44, 1272-1276. doi: $10.1107 / \mathrm{s} 0021889811038970$

Rouse, R. C., Dunn, P. J., Peacor, D. R., and Wang, L. (1998). Structural Studies of the Natural Antimonian Pyrochlores. J. Solid State. Chem. 141, 562-569. doi:10.1006/jssc.1998.8019

Schmitt, A. K., Trumbull, R. B., Dulski, P., and Emmermann, R. (2002). Zr-Nb-REE Mineralization in Peralkaline Granites from the Amis Complex, Brandberg (Namibia): Evidence for Magmatic Pre-enrichment from Melt Inclusions. Econ. Geology 97, 399-413. doi:10.2113/gsecongeo.97.2.399

Subramanian, M. A., Aravamudan, G., and Subba Rao, G. V. (1983). Oxide Pyrochlores - A Review. Prog. Solid State. Chem. 15, 55-143. doi:10.1016/ 0079-6786(83)90001-8

Tindle, A. G., and Breaks, F. W. (1998). Oxide Minerals of the Separation Rapids Rare-Element Granitic Pegmatite Group, Northwestern Ontario. Can. Mineral. 36, 609-635. doi:10.1119/1.879923

van Wambeke, L. (1978). Kalipyrochlore, a New mineral of the Pyrochlore Group. Am. Mineral. 63, 528-530.

Voloshin, A. V., Men'shikov, Yu. P., Pakhomovskiy, Ya. A., and Polezhaeva, L. I. (1981). Cesstibtantite, (Cs,Na)SbTa $\mathrm{O}_{12}$ - a New mineral from Granitic Pegmatites. Zapiski Vsesoyuznoye Mineralogichestogo Obshchestvo 116, 345-351.

Voloshin, A. V., and Pakhomovskiy, Ya. A. (1986). Minerals and mineral Formation Evolution in Amazonite Pegmatites of Kola peninsula. Leningrad: Nauka, 168.

Vorma, A., and Siivola, J. (1967). Sukulaite $-\mathrm{Ta}_{2} \mathrm{Sn}_{2} \mathrm{O}_{7}-$ and Wodginite as Inclusions in Cassiterite in the Granite Pegmatite in Sukula, Tammela, in SW Finland. Bull. de la Comm. géologique de Finlande 229, 173-187.

Williams, P. A., Leverett, P., Sharpe, J. L., Colchester, D. M., and Rankin, J. (2005). Elsmoreite, Cubic $\mathrm{WO}_{3.0} \cdot 5 \mathrm{H}_{2} \mathrm{O}$, a New mineral Species from Elsmore, New South Wales, Australia. Can. Mineral. 43, 1061-1064. doi:10.2113/ gscanmin.43.3.1061

Witzke, T., Steins, M., Doering, T., Schuckmann, W., Wegner, R., and Pöllmann, H. (2011). Fluornatromicrolite, $(\mathrm{Na}, \mathrm{Ca}, \mathrm{Bi})_{2} \mathrm{Ta}_{2} \mathrm{O}_{6} \mathrm{~F}, \mathrm{~A}$ New Mineral Species from Quixaba, Paraiba, Brazil. Can. Mineral. 49, 1105-1110. doi:10.3749/ canmin.49.4.1105

Yang, G., Li, G., Xiong, M., Pan, B., and Yan, C. (2014). Hydroxycalciopyrochlore, a New mineral Species from Sichuan, China. Acta Geologica Sinica - English Edition 88, 748-753. doi:10.1111/1755-6724.12235

Conflict of Interest: The author declares that the research was conducted in the absence of any commercial or financial relationships that could be construed as a potential conflict of interest.

Publisher's Note: All claims expressed in this article are solely those of the authors and do not necessarily represent those of their affiliated organizations, or those of the publisher, the editors and the reviewers. Any product that may be evaluated in this article, or claim that may be made by its manufacturer, is not guaranteed or endorsed by the publisher.

Copyright (c) 2021 Atencio. This is an open-access article distributed under the terms of the Creative Commons Attribution License (CC BY). The use, distribution or reproduction in other forums is permitted, provided the original author(s) and the copyright owner(s) are credited and that the original publication in this journal is cited, in accordance with accepted academic practice. No use, distribution or reproduction is permitted which does not comply with these terms. 